\title{
Effect of Lithium Vapour Shielding on Hydrogen Plasma Parameters
}

\author{
F. Romano ${ }^{1, *}$, P. Rindt ${ }^{2}$, J. Scholten ${ }^{1}$, Y.Hayashi ${ }^{3}$, T.W. Morgan ${ }^{1,2}$
}

${ }^{1}$ DIFFER-Dutch Institute for Fundamental Energy Research, De Zaale 20, 5612AJ Eindhoven, the Netherlands

${ }^{2}$ Department of Applied Physics, Eindhoven University of Technology, Groene Loper 19, 5612 AP Eindhoven, The Netherlands

${ }^{3}$ National Institute for Fusion Science, National Institutes of National Sciences, Toki 509-5292, Japan

*Email: f.romano@,differ.nl

\begin{abstract}
A liquid Li vapour-box divertor is an attractive heat exhaust solution for future fusion reactors. Previous works have established the ability of vapour shielding to protect the wall, but it has not been possible to directly determine the effects of Li vapour on the plasma parameters. Experiments to investigate this were carried out in Magnum-PSI, which is able to generate a plasma with DEMO-divertor relevant conditions. $3 D$ printed tungsten capillary porous structures filled with Li have been used as targets. A reciprocating Langmuir probe was used to determine electron temperature and density close to the target, while the power reduction to the coolant due to vapour shielding was increased from $0 \%$ to $50 \%$. The Langmuir probe measurements directly determined an increase of density by up to 50\% while electron temperature could be inferred to have dropped by up to $33 \%$ compared to the solid target reference case.
\end{abstract}

\section{Introduction}

Among the many challenges to make fusion energy available, one of the most demanding is the handling of the exhaust heat inside a nuclear fusion device. The current solid tungsten configuration has some critical issues, particularly the limited expected lifetime in DEMO Error! Reference source not found., due to problems like erosion, that could represent a showstopper for the fusion project. A solution for this must be found, and one of the most interesting and promising concepts involves the use of a liquid metal (LM) in the divertor to face the incoming plasma power. This will protect the underlying solid tungsten matrix, reducing erosion and thermomechanical stresses. A key feature that comes with the use of a LM surface is the so called vapour shielding (VS) effect. The plasma heating leads to strong heating of the $\mathrm{LM}$, that in turn will leads to the development of an evaporated vapor a cloud in front of the target shielding it from further plasma exposure. The evaporated atoms thus interact with the plasma, cooling it mainly through momentum exchange and radiation, inducing detachment. The removed amount of power will finally be spread over a wider area, reducing the load of $\mathrm{MW} / \mathrm{m}^{2}$ carried to the divertor's strike point Error! Reference source not found.

This design forms an attractive operational mode for the fusion reactor divertor if the LMs are used in a so-called vapor box divertor Error! Reference source not found.Error! 
Reference source not found. because it is capable of naturally inducing and controlling the detachment front while avoiding excessive LMs flow towards the core of the plasma, which would lead to a reduction in fusion power through increased core radiation or fuel dilution Error! Reference source not found.Error! Reference source not found.

The choice of the LM to be used takes an important role as well since each of them has different properties when interacting with different plasma species. Among them, two of the most promising are lithium and tin or even an alloy of both. Other candidates also exist but are less attractive; for example gallium, as it is more corrosive than $\mathrm{Sn}$ or $\mathrm{Li}$ to structural materials. A wider and well detailed list could be found in Error! Reference source not found.

The LM used in this experimental campaign is lithium. This element is attractive as a choice for a vapor box divertor because it can be tolerated in relatively large quantities in the plasma core without reaching excessive dilution rates Error! Reference source not found.. While not typically considered a good radiator, in the low temperature area of the divertor it can be effective, particularly under the non-coronal conditions which are expected to apply in this region Error! Reference source not found.. Li can form chemical bond with hydrogen up to stoichiometric ratio 1:1 helping the remove of exhaust unreacted plasma atoms [ref Baldwin paper]. Because of this a vapor box divertor is considered to have a tritium flow and extraction system to quickly recover the tritium from the liquid and return it to the inner fuel cycle Error! Reference source not found.

Magnum-PSI Error! Reference source not

found. has been used to study the Li VS regime Error! Reference source not found. due to its ability to recreate the detached plasma conditions expected in future divertors, but until now it was not possible to directly infer the influence of VS on the plasma parameters of electron temperature $\left(T_{e}\right)$ and density $\left(n_{e}\right)$ due to risk of damage to the Thomson Scattering (TS) optics while Li is used. Here a reciprocating Langmuir Probe (LP) was used to investigate these parameters during Li VS to identify how the plasma is modified by the VS cloud.

\section{Methods}

\subsection{Magnum-PSI}

Experiments were carried out in Magnum-PSI Error! Reference source not found., which generates a steady-state linear plasma with reactor detachment-like high $n_{e}$ and low $T_{e}$. A scan in power has been made by changing the source current between130-190 A at fixed hydrogen gas flow rate and keeping the magnetic field $\mathrm{B}$ constant at $0.7 \mathrm{~T}$.

$n_{e}$ and $T_{e}$ were determined from TS using a solid Molybdenum reference target. The plasma power thus obtained ranges from $q_{r e f}=5$ to $21 \mathrm{MW} / \mathrm{m}^{2}$, where $q_{r e f}$ is the peak heat flux calculated to arrive at the target surface from the TS data from the Bohm criterion as in Error! Reference source not found.. This therefore ranges from conditions where VS is not expected yet to the full VS regime Error! Reference source not found.. Each shot was $200 \mathrm{~s}$ long to reach the cooling water thermal equilibrium for calorimetry analysis.

\subsection{Targets}

To avoid LM motion due to $J \times B$ forces induced by the superconducting magnetic coils, lithium has been held in place in the target using 3D-printed Capillary Porous Structures (CPSs) made of tungsten as described here Error! Reference source not found.. Each CPS target was filled with around $1.8 \mathrm{~g}$ of $\mathrm{Li}$, and it was ensured that at all times sufficient $\mathrm{Li}$ was present to avoid $\mathrm{Li}$ depletion which could have affected the results.

A helium plasma cleaning shot was performed before the actual experiment which removed any oxide and impurities layers on the surface of the Li-CPS

A solid Mo target with identical geometry has been used as reference under the same plasma conditions.

\subsection{Diagnostics}


Thomson Scattering (TS) Error! Reference source not found. was performed during reference shots to determine the plasma parameters to compare to the lithium cases. The TS laser beam is located $20 \mathrm{~mm}$ in front of the target. Due to the location of the laser port below the target holder, this was covered with a metal shield during $\mathrm{Li}$ exposures to prevent LM droplets falling directly onto the optics, which could lead to damage.

To overcome this limitation, a reciprocating Langmuir Probe previously used in MagnumPSI Error! Reference source not found.Error! Reference source not found. has been used for the first time to assess plasma parameters in VS regime. The probe also enters the plasma at a distance of $20 \mathrm{~mm}$ from the target to be consistent with TS readings, and consists of up to 4 filaments of tungsten encased in an alumina rod and protruding from the latter by $0,5 \mathrm{~mm}$. the alumina tube has a diameter of $4 \mathrm{~mm}$ while each pin is $0,5 \mathrm{~mm}$ in diameter.

Figure 1 contains a representation of the experimental set-up: the plasma coming from the left hits the Li-CPS on the right inducing the vaporization on lithium. In front of the target with a distance of $20 \mathrm{~mm}$ there is the reciprocating LP coming from the back and the optical view of the TS system from the bottom which investigate the same region of the plasma. The total power to the target is determined from the cooling water calorimetry.

The LP was used in single probe mode with voltage sweep from +15 to $-60 \mathrm{~V}$ except for the highest source current cases where the negative voltage amplitude was limited to -45 $\mathrm{V}$ in order to prevent arching between probe's pin, most likely caused by the LM atoms into the plasma which create occasional shortcircuit between the probe's pins.

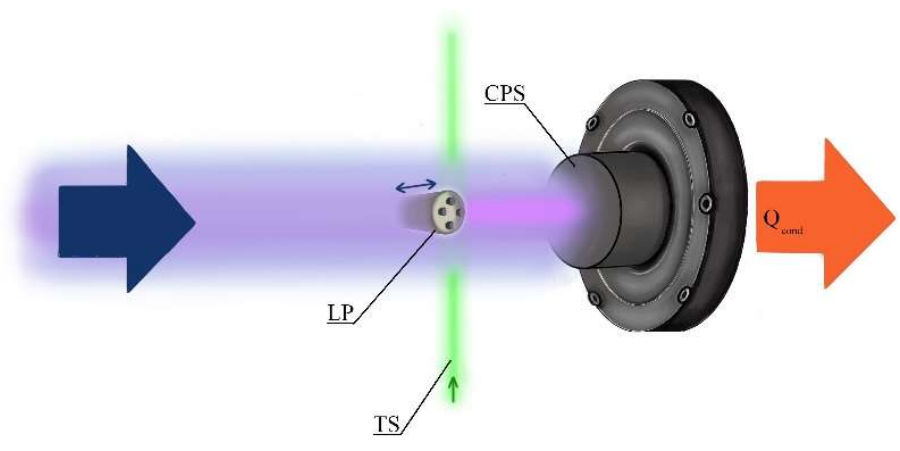

Figure 1 Langmuir probe experimental setup

The LP measurement cycle frequency was 200 $\mathrm{Hz}$ with a sampling rate of $5 \mathrm{MHz}$. To avoid damaging the probe, its duration in the plasma was limited to $0.2 \mathrm{~s}$. Due to the plasma's Gaussian profile, collected current increases as the probe get close to the center, so its movement was deliberately chosen to extend beyond this point, which ensures that the plasma beam center was correctly identified whenever the reading returned two peaks Calorimetry was performed on the cooling water system behind the CPS target holder.

\section{Results}

\subsection{Langmuir Probe}

A comparison between TS and LP was first carried out on the reference sample. Results confirmed the reliability of the LP in obtaining $n_{e}$ data, whereas for $T_{e}$ the values were much higher for the LP than for TS (Figure 2). 


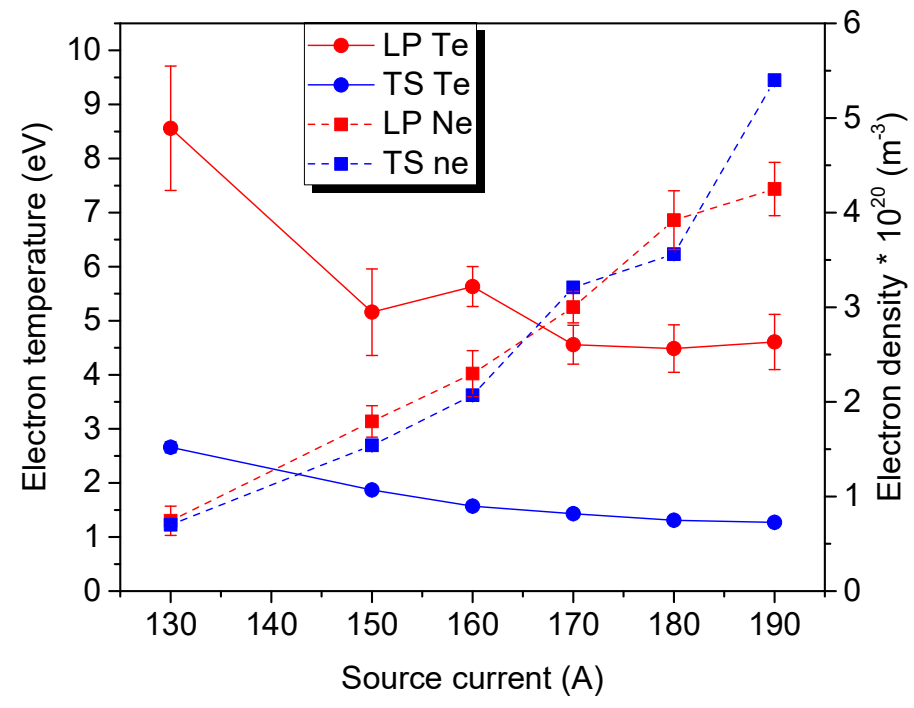

Figure 2 Plasma parameters on reference target acquired with TS and LP both. Solid lines for $T_{e}$, dash lines for $n_{e}$.

This effect has been attributed to fluctuations in density and potential on the timescale of the I-V sweep of the LP Error! Reference source not found.. Therefore only $n_{e}$ data was considered reliable for the measurements during VS. These are shown in Figure 3. As expected, plasma density increases due to interactions between the neutral lithium cloud and the plasma Error! Reference source not found.

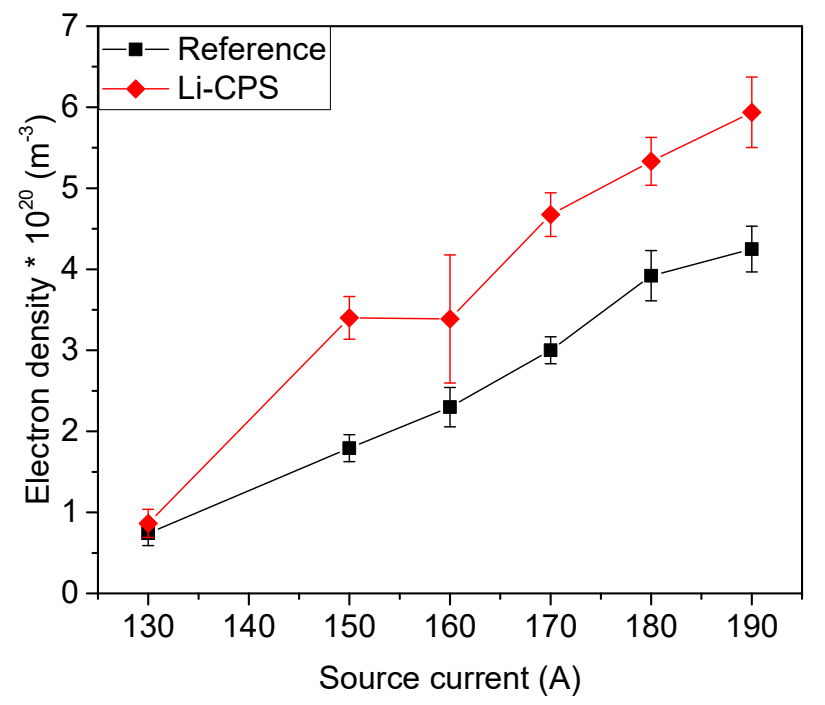

Figure 3 Electron density LP acquired on reference and Li-CPS

\subsection{Calorimetry and $T_{e}$}

To bypass the lack of $T_{e}$ LP data, calorimetry has been used to have an estimation of the power delivered to the target cooling system.

Since TS and LP investigate the plasma in the same position, the reference plasma power and cooling water $\Delta T$ were used together with the Li-CPS cooling water $\Delta T$ to calculate the amount of dissipated power due the lithium presence, as shown in Figure 4. It was therefore possible to estimate the removed plasma power flux to the target after the interaction with the lithium cloud as

$\Delta Q=Q_{\text {cond }}^{r e f}-Q_{\text {cond }}^{L i}=Q_{\text {evap }}^{L i}+Q_{\text {cool }}^{L i}$

Where $Q_{\text {cond }}^{r e f}$ and $Q_{\text {cond }}^{L i}$ are the power flux delivered to the cooling water assuming identical plasma widths in both cases, and $Q_{\text {evap }}^{L i}$ and $Q_{\text {cool }}^{L i}$ the contributions from Latent heat of evaporation plus the cooling processes such as radiation, charge exchange and recombination in the plasma respectively.

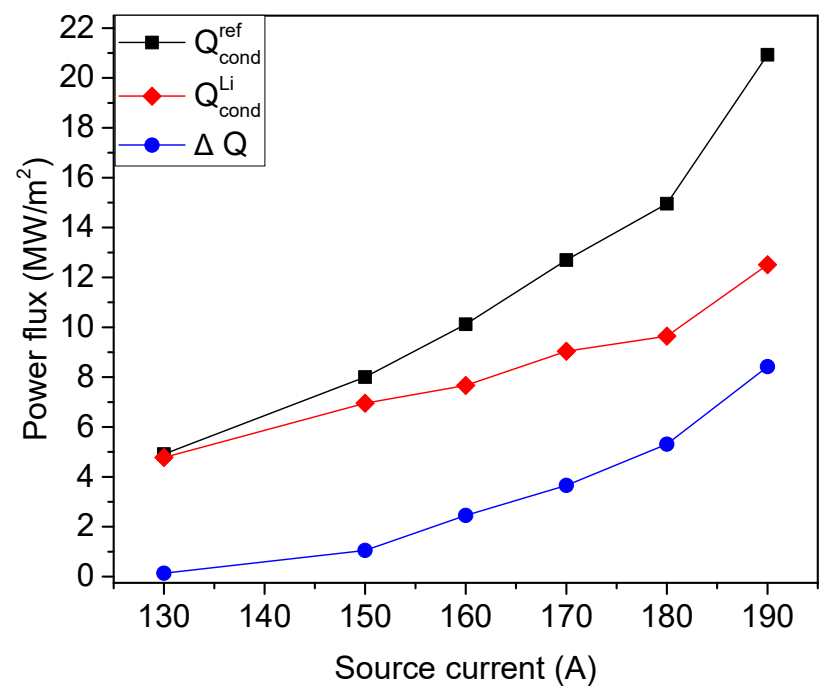

Figure 4 Plasma Power flux removed by the cooling water. The difference in power between the case without lithium and the case with it is shown in blue circles showing the increase with plasma power.

From the measured $n_{e}$ and calorimetric data it is possible to find the expected range of $T_{e}$ from the two extreme cases. In the first case we assume lithium re-deposition rate $R=0$, and that therefore all Li escapes the plasma beam. We also assume that $\Delta Q=Q_{\text {evap }}^{L i}$ so that power 
lost in from the plasma beam is negligible and therefore $q=q_{\text {ref }}$. In the second case we assume $R=1$, i.e. that the lithium is all either ionized or driven by friction force back to the target. In such a case we assume $\Delta Q=Q_{\text {cool }}^{L i}$ and that therefore $q=q_{\text {ref }}\left(Q_{\text {cond }}^{L i} / Q_{\text {cond }}^{\text {ref }}\right)$ and that the increased density arises also from the ionization of $\mathrm{Li}$, which therefore modifies the average mass of the plasma ions.

In both cases we assume that pressure is conserved between the TS and LP measurement region and the sheath entrance. By doing so is possible to get two curves that delimit the region where the real value lies. The two curves and the reference case measured by TS are shown in Figure 5. $R$ was not measured in this experiment but previous results have estimated it to be around 0.9 in similar experiments Error! Reference source not found.-Error! Reference source not found., thus suggesting a value closer to the second case.

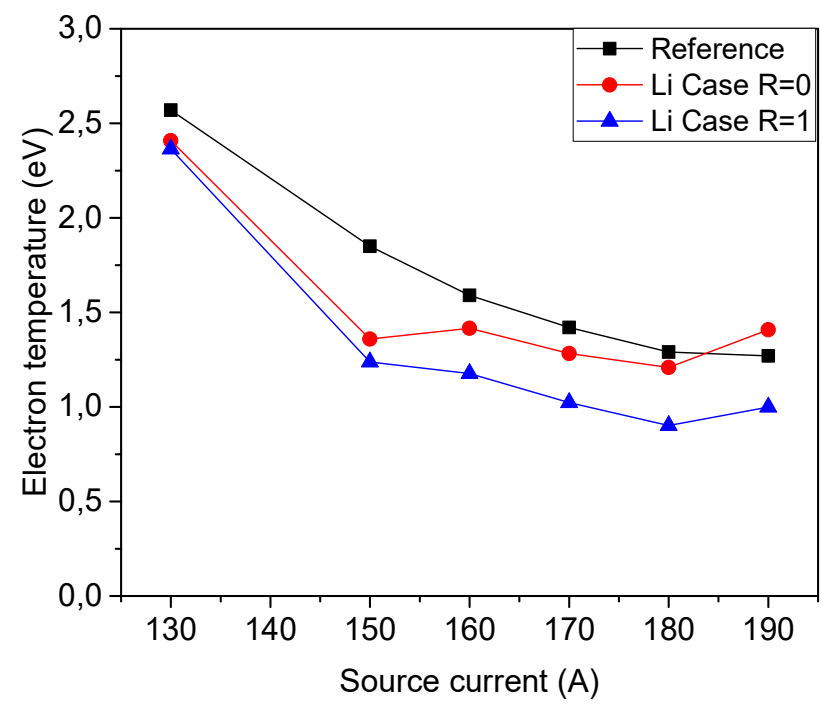

Figure 5 Plasma $T_{e}$. Black curve shows shot on reference target, red on Li-CPS assuming $R=0$, blue on Li-CPS assuming $R=1$

\section{Discussion and conclusion}

By combining the data from the LP, the TS reference data and the calorimetry, it is possible to better estimate the plasma parameters and gain better insight into the VS process. When comparing the reference measurements to the VS measurements, an increase in $n_{e}$ by up to $50 \%$ and a decrease in $T_{e}$ by up to $33 \%$ is indicated. An increase of $n_{e}$ and a decrease in $T_{e}$ is consistent with observations using Sn for VS in Error! Reference source not found.. This cooling is indicative of a combination of collisions slowing the plasma down and radiative processes, while recombination can also start to play an important role, all of which could strongly reduce the power to the strikepoint locations in a VS divertor of a fusion reactor Error! Reference source not found.. This information gives further insight into how a vapor box divertor can be designed and can be expected to perform. Furthermore, the difficulties in the use a LM inside of MagnumPSI and the maintenance that must be done after, stress once again the importance of a vapour box divertor design to prevent lithium escaping toward the plasma core in a nuclear fusion facility, where this kind of problem become even more severe.

\section{Acknowledgements}

DIFFER is part of the institutes organization of NWO. We acknowledge the support of the Magnum-PSI Facility Team at DIFFER. The Magnum-PSI facility at DIFFER has been funded by the Netherlands Organisation for Scientific Research (NWO) and EURATOM. This work has been carried out within the framework of the EUROfusion Consortium and has received funding from the Euratom research and training programme 2014-2018 and 2019-2020 under grant agreement No 633053. The views and opinions expressed herein do not necessarily reflect those of the European Commission.

\section{Data Replication}

The data that support the findings of this study are available at http://doi.org/10.5281/zenodo.4897758

\section{References}

[1] Morgan TWet al 2017 Liquid metals as a divertor plasma-facing material explored using 
the Pilot-PSI and Magnum-PSI linear devices Plasma Phys. Control. Fusion 60014025

[2] Van EdenGGet al 2016 Self-regulated plasma heat flux mitigation due to liquid $\mathrm{Sn}$ vapor shielding Phys. Rev. Lett. 116135002

[3] Goldston R J, Myers R and Schwartz J 2016 The lithium vapor box divertor Phys. Scr. 2016 T167 014017

[4] Emdee EDet al 2019 A simplified lithium vapor box divertor Nucl. Fusion 59086043

[5] Rognlien TDet al 2019 Simulations of a high-density, highly-radiating lithium divertor Nuclear Materials and Energy 18 233-8

[6] Emdee EDet al 2021 Predictive modeling of a lithium vapor box divertor in NSTX-U using SOLPS-ITER Nuclear Materials and Energy 27101004

[7] Mirnov S 2009 Plasma-wall interactions and plasma behaviour in fusion devices with liquid lithium plasma facing components $\mathrm{J}$. Nucl. Mater. 390 876-85

[8] BaldwinMJ et al 2002 Deuterium retention in liquid lithium Nuclear Fusion 42 1318-23

[9] Humrickhouse PWet al 2019 The impacts of liquid metal plasma-facing components on fusion reactor safety and tritium management Fusion Sci. Technol. 75 973-1001

[10] Van EckHJNet al 2019 High-fluence and high-flux performance characteristics of the superconducting magnum-PSI linear plasma facility Fusion Eng. Des. 142

[11] Rindt P et al 2019 Power handling and vapor shielding of pre-filled lithium divertor targets in Magnum-PSI Nucl. Fusion 59 056003

[12] Stangeby PCet al 2000 The Plasma Boundary of Magnetic Fusion Devices (Philadelphia, Pennsylvania: Institute of Physics Pub) p61

[13] Rindt P et al 2009 Performance of liquidlithium-filled 3D-printed tungsten divertor targets under deuterium loading with ELMlike pulses in Magnum-PSI. Nuclear Fusion, 2021, 61.6: 066026.G J van Rooij et al Plasma Phys. Control. Fusion 51124037

[14] Hayashi Y et al 2016 Plasma detachment study of high density helium plasmas in the pilot-PSI device Nucl. Fusion 56126006

[15] Hayashi Y et al 2019 Application of ion sensitive probe to high density plasmas in magnum-PSI Plasma and Fusion Research 14 1202135

[16] Van EdenGGet al 2017 Oscillatory vapour shielding of liquid metal walls in nuclear fusion devices Nature Communications 8 1-10

[17] Van RooijGJ et al 2009 Plasma Phys. Control. Fusion 51124037

[18] JeškoKet al 2018 Studying divertor relevant plasmas in the pilot-PSI linear plasma device: experiments versus modelling Plasma Phys. Control. Fusion 60125009 